\title{
STATISTICAL ANALYSIS OF ADHESIVE LAYER THICKNESS` DISTRIBUTION ON PERFECT BOUNDED BROCHURES
}

\author{
Magdolna Pál (D), Sandra Dedijer (D), Živko Pavlović (D), Bojan Banjanin (D), Jelena Vasić \\ University of Novi Sad, Faculty of Technical Sciences, \\ Department of Graphic Engineering and Design, Novi Sad, Serbia
}

\begin{abstract}
Perfect binding has become one of the most popular binding methods for low-and mid-range graphic products, but with the appearance of new adhesives and application techniques, it is more and more important for the production of high quality, short-run products too. The influencing factors of perfect binding quality are numerous, involving different parameters and aspects of graphic production. Along the paper's properties, book block parameters and spine preparation technique, the used adhesive and its application could have a direct influence on the paper/adhesive interaction and therefore on the adhesive bond quality, i.e. binding strength, too. In this study, a statistical analysis of overall adhesive layer thickness and its distribution along the book block spine has been performed. This analysis was aiming to investigate the influence of the book block volume and two gluing system set-up parameters, the nominal adhesive thickness and gluing length modification, on the adhesive application consistency. The statistical analysis showed that the book block volume had a significant influence on the obtained results of overall adhesive layer thickness (based on the p-value of 0.05) and the adhesive layer became more evenly applied to the block spine by increasing the book block volume. The influence of the nominal adhesive thickness and the glue length modification were also statistically significant (at level $p<0.05$ ) on the obtained overall adhesive thickness, however, the corresponding post hoc tests showed that not all the observed groups differed significantly from each other. In addition, the obtained results for adhesive distribution along the book block spine showed more uniform application for the higher values of nominal adhesive thickness and glue length modification.
\end{abstract}

Key-words: perfect binding, adhesive layer, book block spine

\section{INTRODUCTION}

Perfect binding is the most frequently used binding techniques for low and mid-range quality brochures with high-volume, primarily because of its suitability for mass production. Also, due to the development and availability of new adhesives, perfect binding has become an important finishing operation for the high-quality and short-run products, too (Kipphan, 2001; Liebau et al, 2001; Pasanec Preprotić et al., 2012). By its growing range of application, the quality control of perfect bounded products gained on importance. The page-pull test (also known as pull test) is the only standardized method available today for the quality assessment of perfect bindings, and it has an essential role in both quality verifications, the process and final control. Along with other test parameters (such as test position, specimen alignment and fixing, etc.) the specimen opening behaviour was identified to have a direct influence on results of page-pull tests (ISO 19594:2017, 2017). Uniform adhesive application can be recognized as a precondition for the adequate opening behaviour of the produced brochures, therefore the adhesive layer thickness and application consistency have particular importance. The desirable amount of adhesive layer thickness mostly depends on the size of the book block. Book blocks with higher volume (i.e. thicker) require a higher amount of adhesive and vice versa, for thinner book blocks thinner adhesive layer is sufficient to obtain the expected binding strength, spine shape stability and opening behaviour. Applying more adhesive than recommended will reduce the book block spine flexibility, making it more difficult to open and lay down flat without destroying the spine shape. Also, the adhesive consumption and overall production costs will increase, but also frequent work stoppage/downtime will slow down the production process due to the excess adhesive accumulation on the binding machines. Thinner adhesive layer than recommended will result in insufficient adhesion and binding strength, decreased book block integrity and reduced adhesive open-time (Lauren, 2017). The uniform adhesive application over the book spine is also fundamental in achieving high adhesive bond strength, the stability of the book block spine shape and adequate brochure opening behaviour. The uneven distribution of adhesive layer could directly affect the overall adhesive bond strength, but also the aesthetic feature of the finished brochure (wavy shape of the book block due to the unwanted local glue accumulation). 
The scope of this investigation is limited to the statistical analysis of overall adhesive layer thickness and its distribution along the book block spine to investigate the influence of book block volume (thickness) and two gluing system set-up parameters, the nominal adhesive thickness and gluing length modification, on the adhesive application consistency.

\section{MATERIALS AND METHODS}

For this investigation the brochures 'book blocks were prepared from commercially available uncoated, wood-free, offset paper with the basis weight of $80 \mathrm{~g} / \mathrm{m}^{2}$ (IQ Print, Mondi). The selected paper has good surface properties and smoothness and it is usually used for graphic products such as annual reports, magazines, books and brochures, printed with sheet-fed or web offset printing technique (Mondigroup, 2016). The brochures' covers were prepared from glossy coated cardboard with a basis weight of $300 \mathrm{~g} / \mathrm{m}^{2}$ (Nevia, n.d.). The binding operation was done on Horizon BQ-270 automated perfect binding machine with hot-melt adhesive Mitol-Termokol Ultra 2410/05 (Grafino, 2017), at working temperature of $170^{\circ} \mathrm{C}$. The binding machine is equipped with a single-clamp, a double rollers gluing unit and a milling section for book block spine processing (Xerox Corporation, 2018).

The dimensions of the prepared brochures were $176 \mathrm{~mm} \times 250 \mathrm{~mm}$ (width $\mathrm{x}$ height), while the book block thickness (the number of sheets, i. e. the volume) has been increased from 50 sheets up to 250 sheets, with the increment of 50 sheets. The specifications of prepared brochure samples (book block volume) and the binding process set-up parameters (nominal adhesive layer thickness and gluing length shortening) are presented in Table 1.

Table 1: Specifications of prepared brochure samples

\begin{tabular}{|c|c|c|c|c|}
\hline $\begin{array}{l}\text { Group } \\
\text { number }\end{array}$ & $\begin{array}{c}\text { Book block } \\
\text { volume } \\
\text { [no. of sheets] }\end{array}$ & $\begin{array}{c}\text { Book block } \\
\text { thickness } \\
{[\mathrm{mm}]}\end{array}$ & $\begin{array}{c}\text { Nominal adhesive } \\
\text { layer thickness } \\
{[\mathrm{mm}]}\end{array}$ & $\begin{array}{c}\text { Glue line } \\
\text { length } \\
\text { shortening } \\
\text { [mm] }\end{array}$ \\
\hline 1. & 50 sheets & 5 & \multirow{5}{*}{$1.8 \mathrm{~mm}$} & \multirow{7}{*}{$-1.5 \mathrm{~mm}$} \\
\hline 2. & 100 sheets & 10 & & \\
\hline 3. & 150 sheets & 15 & & \\
\hline 4. & 200 sheets & 20 & & \\
\hline 5. & 250 sheets & 25 & & \\
\hline 6. & 150 sheets & \multirow{4}{*}{15} & $1.4 \mathrm{~mm}$ & \\
\hline 7. & 150 sheets & & $2.2 \mathrm{~mm}$ & \\
\hline 8. & 150 sheets & & \multirow{2}{*}{$1.8 \mathrm{~mm}$} & $0 \mathrm{~mm}$ \\
\hline 9. & 150 sheets & & & $-3 m m$ \\
\hline
\end{tabular}

To investigate the influence of the observed parameters on the adhesive layer thickness distribution, after 3-side trimming each brochure sample was cut into 24 uniform segments along the book block spine, using a high-speed cutting machine (Perfecta 72 HTVC). The thickness measurement was carried out using a measuring magnifier with metric scale (field of view: 0 to $10 \mathrm{~mm}$, minimum scale division: $0.1 \mathrm{~mm}$ ). Five brochure samples were prepared for each parameter settings and the thickness measurement was repeated three times on one segment, hence 15 measurements were carried out for each segment, or altogether 360 measurements for each parameter.

\section{RESULTS AND DISCUSSION}

The mean values of adhesive layer thickness, their standard deviations and corresponding coefficient of variations were calculated for the entire book block spine (overall values) and for each measuring position (adhesive layer profiles). The obtained results are presented in that manner. In Figure 1a-c the overall mean values and standard deviations are given for each parameter group, while Figures 2-4 are showing the adhesive layer thickness profiles along the book block spine likewise for each parameter group.

As it can be seen in Figure 1a, the overall mean values of adhesive layer thickness have the increasing tendency by increasing the brochures thickness, i.e. volume. For the thinnest brochures the overall mean value of adhesive layer thickness was $0.500 \mathrm{~mm}$, while values of $0.591 \mathrm{~mm}, 0.741 \mathrm{~mm}, 0.863 \mathrm{~mm}$ and $0.894 \mathrm{~mm}$ were delivered for brochures with 10, 15, 20 and $25 \mathrm{~mm}$ thick book blocks, respectively. 

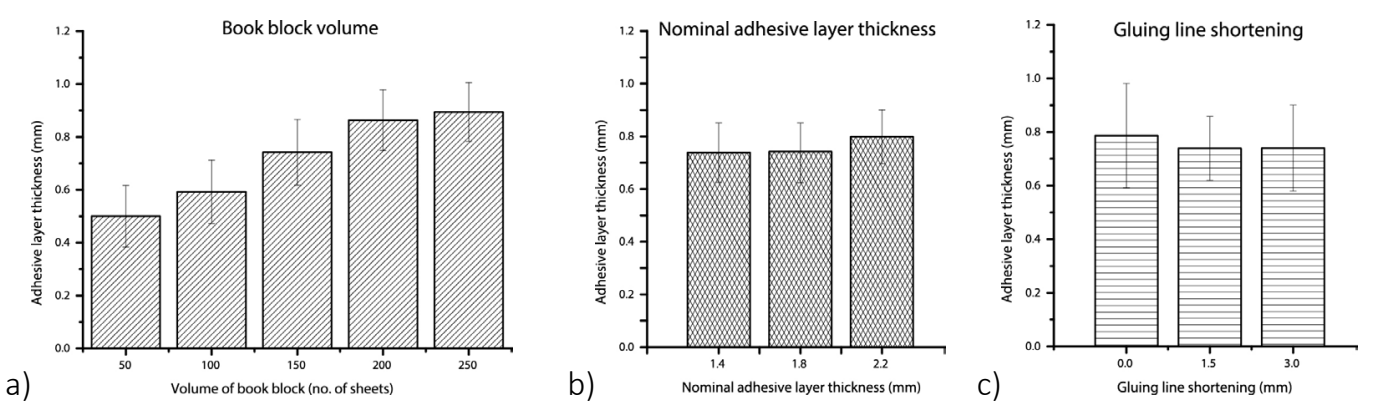

Figure 1: Overall mean values of adhesive layer thicknesses regarding to the book block thickness (a), nominal adhesive layer thickness (b) and gluing line shortening set-up values (c)

Comparing the results by nominal adhesive layer thicknesses (Figure 1b) it can be noticed that the obtained values were very similar, however a slightly increasing tendency is apparent. The mean values were 0.738 and 0.741 for nominal layer thicknesses of $1.4 \mathrm{~mm}$ and $1.8 \mathrm{~mm}$, while for the brochures with the nominal layer thickness of $2.2 \mathrm{~mm}$ it was $0.798 \mathrm{~mm}$. In addition, although the nominal adhesive layer thicknesses have been set much higher, none of the prepared samples had exceeded the adhesive layer thickness of $0.9 \mathrm{~mm}$ over the book block spine. The reason for that is the nominal adhesive thickness on the book block spine corresponds to the maximum adhesive thickness on the gluing rollers, which cannot be entirely transferred to the book block spine. By analysing the results regarding different gluing length values (Figure 1c), the overall mean values of adhesive layer thickness were also very similar, but a slight decreasing tendency can be noticed. With the shortening of 1.5 and $3 \mathrm{~mm}$, the mean thicknesses were 0.741 and 0.740 , while for the samples without gluing length modifications (i.e. with $0 \mathrm{~mm}$ shortening) the mean value of adhesive layer thickness was $0.787 \mathrm{~mm}$.
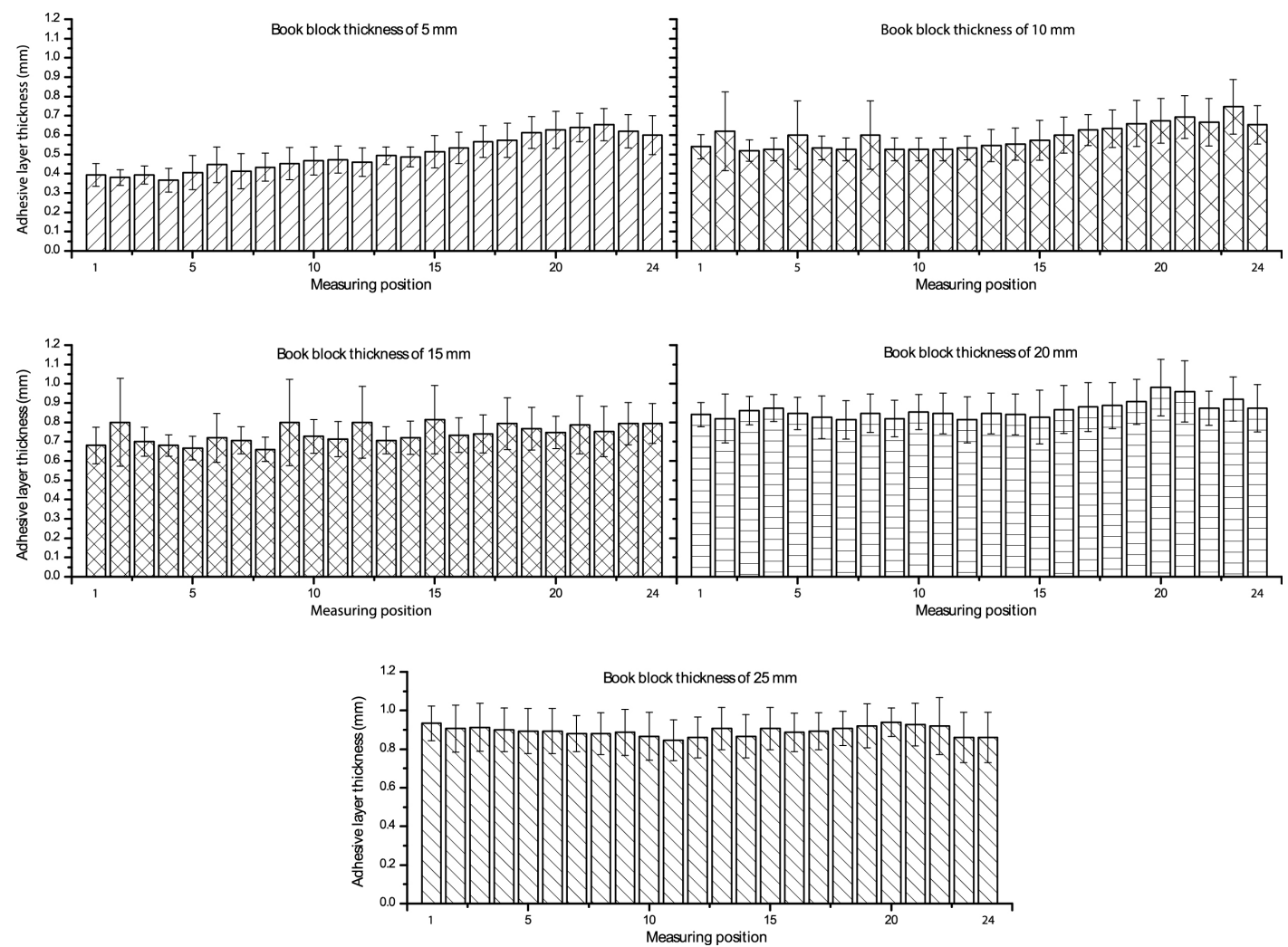

Figure 2: Adhesive layer thickness profiles for brochures with different book block thicknesses

From the adhesive layer profiles for brochures with different book block thicknesses (Figure 2) it can be noticed that in the case of thinner brochures (especially for 5 and $10 \mathrm{~mm}$ thick book blocks) the adhesive layer thickness had visible increasing tendency along the measuring positions, with ranges from $0.367 \mathrm{~mm}$ to $0.653 \mathrm{~mm}$ for book blocks of $5 \mathrm{~mm}$ and $0.520 \mathrm{~mm} \div 0.747 \mathrm{~mm}$ for book blocks of $10 \mathrm{~mm}$. 
Since position 1 corresponds to brochure's upper part, where the gluing process begins and position 24 corresponds to the brochure's bottom part, where the gluing was stopped, these increasing tendencies along the measuring points could indicate the undesirable glue accumulation at the end of the book block spine during the glue application process. For higher book block thicknesses this uneven adhesive application is not that distinct, however slight increasing tendency can also be noticed with ranges from $0.660 \mathrm{~mm}$ to $0.813 \mathrm{~mm}$ for $15 \mathrm{~mm}$ thick book blocks, from $0.813 \mathrm{~mm}$ to $0.980 \mathrm{~mm}$ for $20 \mathrm{~mm}$ thick book blocks and from $0.847 \mathrm{~mm}$ to $0.940 \mathrm{~mm}$ for $25 \mathrm{~mm}$ thick ones. The moderately low values of standard deviation indicate high consistency of the measured values (the ranges of the corresponding coefficient of variations were: $9.27 \% \div 22.15 \%, 10.79 \% \div 29.55 \%, 8.24 \% \div 26.75 \%, 7.52 \% \div 16.79 \%$ and $7.84 \% \div$ $16.02 \%$, for samples with $5,10,15,20$ and $25 \mathrm{~mm}$ thick book block, respectively).
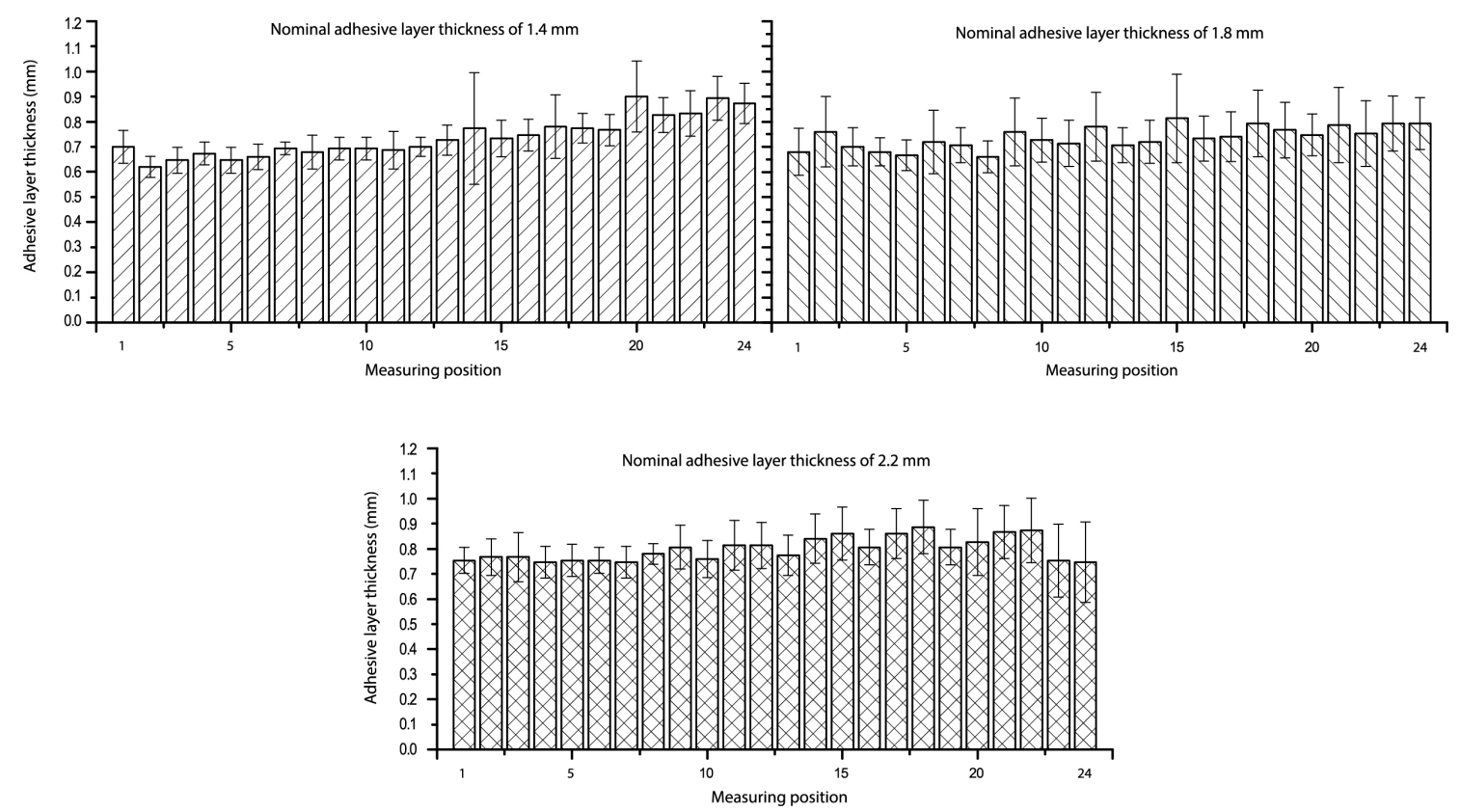

Figure 3: Adhesive layer thickness profiles for brochures with different nominal adhesive layer thicknesses

Comparing the obtained adhesive layer profiles for different nominal adhesive layer thicknesses (Figure 3), a mild increasing tendency can be observed along the measuring positions (from position 1 to position 24), although the overall mean values were very similar. These tendencies are more visible for the lowest nominal adhesive layer thickness value $(1.4 \mathrm{~mm})$, than for the other two nominal thicknesses. The ranges of obtained results were $0.620 \mathrm{~mm} \div 0.900 \mathrm{~mm}, 0.660 \mathrm{~mm} \div 0.813 \mathrm{~mm}$ and $0.747 \mathrm{~mm} \div 0.887 \mathrm{~mm}$ for nominal adhesive thicknesses of $1.4 \mathrm{~mm}, 1.8 \mathrm{~mm}$ and $2.2 \mathrm{~mm}$, respectively.

By analysing the adhesive layer profiles regarding different gluing length values (Figure 4) a certain waviness of the adhesive layer can be noticed on the samples with no glue line shortening (set-up value of $0 \mathrm{~mm}$ ). This waviness was most likely caused by the excessive adhesive application on the book block spine, especially at the beginning of the gluing process. The brochures with $1.5 \mathrm{~mm}$ shortening on both ends of the book block had more evenly spread adhesive layer, but the most uniform adhesive layer was achieved with the shortest gluing application $(-3 \mathrm{~mm})$ since there were no redundant adhesives at the ends of the brochures. The measured thickness values for both sample groups with shortened gluing line were in very similar range $(0.66 \mathrm{~mm} \div 0.813 \mathrm{~mm}$ for $-1.5 \mathrm{~mm}$, and $0.673 \mathrm{~mm} \div$ $0.800 \mathrm{~mm}$ for $3 \mathrm{~mm}$ ), while samples without gluing length modifications had higher maximum values (range $0.673 \mathrm{~mm} \div 0.960 \mathrm{~mm}$ ) and more visible increasing tendency along the measuring positions. 

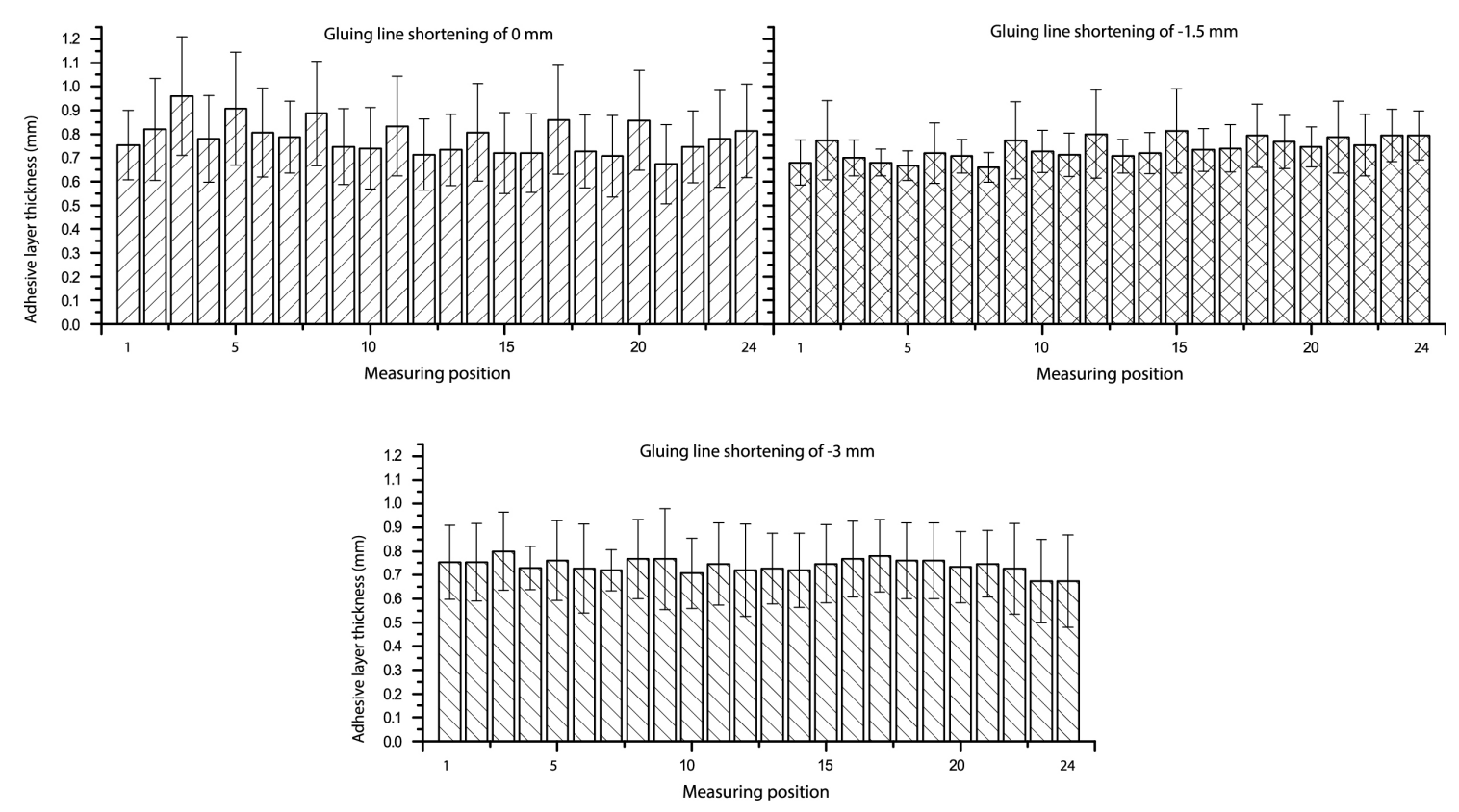

Figure 4: Adhesive layer thickness profiles for brochures with different gluing length

Although the presented graphs and profiles delivered useful information about the changes in applied adhesive layer thicknesses, they do not set out whether these changes are statistically significant. In order to determine the statistical significance of the differences in the obtained mean values of adhesive layer thicknesses regarding the observed parameter groups, detailed statistical analyses were done by one-way ANOVA and corresponding post hoc tests (Dunnett's T3). Besides the statistical significance delivered by ANOVA, the effect size (the practical significance) is also calculated for each sample group using partial eta squared. According to Cohen, the value of 0.01 is classified as a small effect, 0.06 as a medium effect, and 0.14 as a large effect (Pallant, 2005). Along ANOVA, where it was applicable, the correlation analysis was used to describe the strength and direction of the linear relationship between the measured adhesive layer thickness and measuring points. Preliminary analyses were performed to ensure no violation of the assumptions of normality, linearity, homogeneity (for ANOVA) and homoscedasticity (for correlation analysis). All the statistical analyses were done using IBM SPSS statistics software (version 20), with a significance level of $p<0.05$ for ANOVA and $p<0.01$ for correlation analysis.

Based on the results of ANOVA, in the case of book block volume, there is a statistically significant difference in mean values of the adhesive layer thicknesses with large effect size $[F(4,1795)=759.643$, $p=0.000$, partial eta squared $=0.628$ ]. Post hoc comparisons using the Dunnett's $T 3$ test showed that all the mean values of adhesive layer thicknesses regarding to the different book block volume differ significantly $(M=0.500, S D=0.116 ; M=0.591, S D=0.119 ; M=741, S D=0.124 ; M=0.863, S D=0.115$ and $\mathrm{M}=0.894, \mathrm{SD}=0.111$ ). Results of one-way ANOVA for the nominal adhesive layer thickness showed that there was a statistically significant difference in mean values of the adhesive layer thicknesses, $[F(2,1077)=32.369, p=0.000]$, but with small effect size according to Cohen (partial eta squared $=0.057$ ). The post hoc comparisons (Dunnett's T3) indicated that only the highest nominal adhesive layer thickness $(2.2 \mathrm{~mm})$ resulted in significantly different mean value $(M=0.798, S D=0.102)$ comparing the other two, lower set-up values $(M=0.738, S D=0.112$ for samples with nominal adhesive layer thickness of $1.4 \mathrm{~mm}$ and $\mathrm{M}=0.741, \mathrm{SD}=0.124$ for samples with $1.8 \mathrm{~mm}$ thick nominal adhesive layer set-up). The mean values for the two lower thickness set up did not differ significantly. Regarding the gluing line shortening set-up, the results of one-way ANOVA have shown a statistically significant difference between the observed groups $[F(2,1077)=9.892, p=0.000]$, but with small effect size (partial eta squared $=0.018$ ) like in the case of nominal adhesive layer thickness set-up. Based on the corresponding post hoc test (Dunnett's T3) it has been found that only the samples with no gluing line shortening gave statistically significant different mean value $(M=0.787, S D=0.195)$ comparing the samples with glue line shortening of $1.5 \mathrm{~mm}$ and $3 \mathrm{~mm}$ $(M=0.741, S D=0.124$ and $M=0.740, S D=0.161$, respectively). The mean values of the two modified gluing length's groups did not differ significantly.

The results of conducted correlation analysis have shown a positive correlation between the measuring positions and the adhesive layer thickness in most of the observed parameters, however strong 
correlations were registered only in two cases: for the book block thickness of $5 \mathrm{~mm}(\mathrm{r}=0.742, \mathrm{n}=360$, $p<0.05)$ and nominal adhesive layer thickness of $1.4 \mathrm{~mm}(r=0.637, n=360, p<0.05)$. These results confirm the previously observed tendencies presented in Figure 2 and 3 , that during the glue application process undesirable glue accumulation is happening at the end of the book block spine. For higher values of these parameters, the correlation coefficient (Pearson's $r$ ) values were much lower, indicating medium ( $r=0.416$ for brochures with $10 \mathrm{~mm}$ thick book block) and small correlations ( $r=0.216$ and $r=0.234$ for the book blocks of 15 and $20 \mathrm{~mm}$, respectively; $r=0.216$ and $r=0.242$ for the brochures with 1.8 and $2.2 \mathrm{~mm}$ thick nominal adhesive layer set-up) or no correlations at all ( $r=-0.009$ for $25 \mathrm{~mm}$ thick book blocks). In the case of glue length modification, the results of correlation analysis were not as consistent as for the other two parameters, showing in one case medium positive correlation between the measuring positions and the adhesive layer thickness ( $r=0.216$ for glue line shortening of $1.5 \mathrm{~mm})$, in other small negative $(r=-0.129$ for samples with no gluing length modification) and in the third no correlations at all ( $r=-0.074$ for glue line shortening of $3 \mathrm{~mm}$ ).

\section{CONCLUSIONS}

In this paper, the influence of the book block volume and two gluing system set up parameters (nominal adhesive layer thickness and gluing length) on the overall adhesive layer thickness and its distribution along the book block spine has been analysed and presented. Based on the obtained results for overall mean values of adhesive layer thickness it can be conducted that it has a notable increasing tendency by increasing the brochures volume (i.e. thickness). For the nominal adhesive layer thickness, the same increasing tendency was fairly visible, as the decreasing tendency for the gluing length modification. These remarks are confirmed by the performed statistical analysis, one-way ANOVA and the corresponding post hoc tests. The obtained results of these tests imply that changes in brochure book block volume influence significant changes in adhesive layer thicknesses for all observed book block volumes (50-250 sheets). In case of two gluing system set-up parameters, the nominal adhesive layer thickness and the gluing length modification, significant differences were observed in the adhesive layer thickness values, but only for the higher amount of glue application (for the nominal thickness of $2.2 \mathrm{~mm}$ and with no gluing length modification). Regarding the adhesive layer distribution along the book block spine, the results of correlation analysis showed that the adhesive layer became more uniformly applied by increasing the book block volume, but also for higher values of nominal adhesive thickness and the shortest length of glue application.

\section{ACKNOWLEDGMENTS}

This work was supported by the Serbian Ministry of Science and Technological Development, Grant No.: 35027 "The development of the software model for improvement of knowledge and production in the graphic arts industry".

\section{REFERENCES}

[1] Grafino: "Technical data for Termokol Ultra 2410/05 hot-melt adhesive", URL: http://www.grafino.rs/proizvodi/graficki-lepkovi/\%201.2./, (last request: 2017-08-25).

[2] International Organization for Standardization, ISO 19594:2017: “Graphic technology -Test method for the determination of the binding strength for perfect-bound products - Page-pull test working upwards", International Organization for Standardization, 2017.

[3] Kipphan, H.: "Handbook of Print Media: Technologies and Production Methods", (Springer-Verlag, Heidelberg, 2001.). doi: 10.1007/978-3-540-29900-4

[4] Lauren, O.: "How Spine Glue Thickness Can Affect Bookbinding Integrity", URL: http://rsindustrial.com/blog/glue-thickness-bookbinding-integrity/, (last request: 2018-03-10).

[5] Liebau, D.; Heinze, I.: "Industrielle Buchbinderei” (Verlag Beruf+Schule, Paderborn, 2001.)

[6] Mondigroup: "IQ Print: your reliable and flexible uncoated wood-free paper for offset printing", URL: https://www.mondigroup.com/en/products-and-solutions/categories/office-andprofessional-printing-papers/iq-print/, (last request: 2017-08-28). 
[7] Nevia: "Technical data Nevia C2S". URL:

https://static1.squarespace.com/static/56ab875f0ab3777445d9785e/t/57334e5759827ee074bc3 657/1462980183566/CathayNevia_C2SNew.pdf, (last request: 2017-08-25).

[8] Pallant, J.: "A step by step guide to data analysis using SPSS for Windows (Version 12)", (Allen \& Unwin, Ligare, Sydney, 2005.).

[9] Pasanec Preprotić, S., Babić, D., Tuzović, A.: "Research on adhesive joint strength dependency on loose leaf position in a text block", Tehnicki vjesnik-Technical Gazette, 19(1), 43-49, 2012.

[10] Xerox Corporation: "Technical data for Horizon BQ 270", URL: https://www.xerox.com/digitalprinting/feeders-print-finishing/offline-finishers/horizon-bq270-perfect-binder/enus.html/, (last request: 2018-01-28).

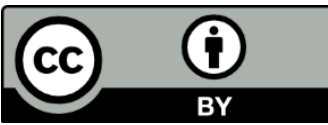

(C) 2018 Authors. Published by the University of Novi Sad, Faculty of Technical Sciences, Department of Graphic Engineering and Design. This article is an open access article distributed under the terms and conditions of the Creative Commons Attribution license 3.0 Serbia (http://creativecommons.org/licenses/by/3.0/rs/). 